\section{Jahre Schiffbruch im Handgelenk}

$\mathrm{E}^{\mathrm{n}}$ nde der 1990er-Jahre fehlte bei Haus- ärzten wie auch Orthopäden noch das Bewusstsein für die Probleme eines Kahnbeinbruchs und seiner Folgen. Über die Hälfte aller Betroffenen wurden entweder nicht ausreichend diagnostiziert oder nicht konsequent behandelt und entwickelten schlussendlich eine Skaphoidpseudarthrose.

Zunächst standen für die Diagnostik lediglich konventionelle Röntgenaufnahmen des Handgelenks zur Verfügung. Da eine Fraktur bei einer Bildgebung in zwei Ebenen oft nicht erkennbar war, behalf man sich mit gehaltenen Aufnahmen wie dem Skaphoid-Quartett oder Schrägaufnahmen wie der Stecheraufnahme. Bei entsprechender Klinik und fehlendem radiologischen Frakturnachweis erfolgte eine Ruhigstellung für zwei Wochen; danach wurden die Röntgenaufnahmen in der Hoffnung auf die Darstellbarkeit einer Sklerosierunglinie wiederholt. Heute gilt die DünnschichtCT als Goldstandard der Diagnostik.

Die konservative Therapie bestand initial in einer Ruhigstellung im Oberarm- gips für bis zu zwölf Wochen. Auch hier kam es regelmäßig zur Ausbildung einer Pseudarthrose.

Mit dem Verständnis, dass zur Heilung einer Skaphoidfraktur eine Fragmentkompression erforderlich ist, begannen die ersten Therapieversuche mittels Schraubenosteosynthese. Besonders problematisch war hierbei die anatomische Form des Skaphoids sowie ihre Nähe zum STT-Gelenk und die daraus resultierende schwierige komplett intraossäre Positionierung einer Schraube. Zunächst wurden Zielgeräte, die unter Durchleuchtung eingebracht wurden eingesetzt. Dies erforderte jedoch einen relativ großen operativen Zugangsweg. Nach Einführung der Herbertschraube, die eine Kompression auf die Fragmente mittels zweier unterschiedlicher Gewindesteigungen bewerkstelligt, erfolgte relativ kurz danach die Entwicklung kanülierter Schraubentypen. Diese markierten den Endpunkt des Entwicklungsprozesses und erlaubten eine Osteosynthese ohne die Notwendigkeit, die Handgelenkskapsel zu eröffnen.

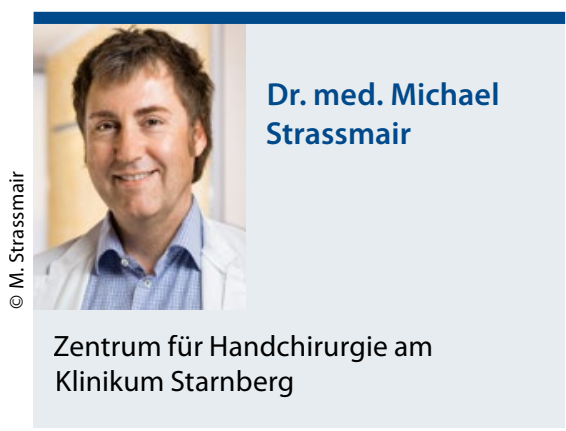

In den vergangenen Jahren erfolgte eine Optimierung der kanülierten Schrauben. Im Vordergrund stand hierbei die Verkleinerung des Schraubendurchmessers, um nach dem Prinzip „Steel can't heal“ möglichst viel Knochenmasse für den Heilungsprozess zu erhalten. Durch selbstschneidende Schraubenköpfe entfiel außerdem die biologisch ungünstige Hitzeentwicklung durch das Vorbohren des Schraubenlagers.

Für besondere Indikationen, beispielsweise für sehr junge Patienten, stehen heute inzwischen auch resorbierbare Implantate zur Verfügung.

\title{
Knieendoprothetik: Was ist heute anders?
}

D ie Knieendoprothetik hat in den vergangenen 20 Jahren eine erhebliche Entwicklung durchlaufen. Im Laufe der 1990er-Jahren wurde die Kinematik des Kniegelenks immer besser verstanden. Die Vielzahl unterschiedlicher Systeme und Philosophien der endoprothetischen Versorgung des Kniegelenks spiegelte die Schwierigkeiten wider, die bei der technischen Umsetzung der neuen Erkenntnisse auftraten. Die Entwicklung ging hin zum weitgehenden Erhalt der physiologischen Gelenkkinematik durch eine knochensparende und den anatomischen Verhältnissen nachempfundene Form der OberflächenersatzProthesen mit der zunehmenden Option modularer Systemerweiterungen.

Neben der moderneren Philosophie und Medizintechnik hat sich auch die Operationstechnik gewandelt. Zur Reduzierung der Komorbidität durch den für die Implantation einer Endoprothe- se notwendigen operativen Zugang haben sich verschiedene Verfahren der minimalinvasiven Chirurgie etabliert. Die Ziele sind ein verringertes Operationstrauma unter Erhalt der Propriozeption, eine verkürzte Operationsdauer mit geringerem Blutverlust, eine schnellere unmittelbar postoperative Rehabilitation und eine kürzere Hospitalisierung.

In den Händen eines erfahrenen Knieoperateurs ist die Implantation einer Endoprothese heute eine komplikationsarme, erfolgssichere Operation. Gerade die minimalinvasive Technik verlangt dem Operateur jedoch ein Höchstmaß an Training und operationstechnischer Präzision ab.

Nachdem die Möglichkeiten der Optimierung der Werkstoffe und des Prothesendesigns derzeit relativ weit ausgeschöpft scheinen, steht zur weiteren Qualitätssteigerung die Fortbildung der Operateure im Fokus. Seit 2006 wird

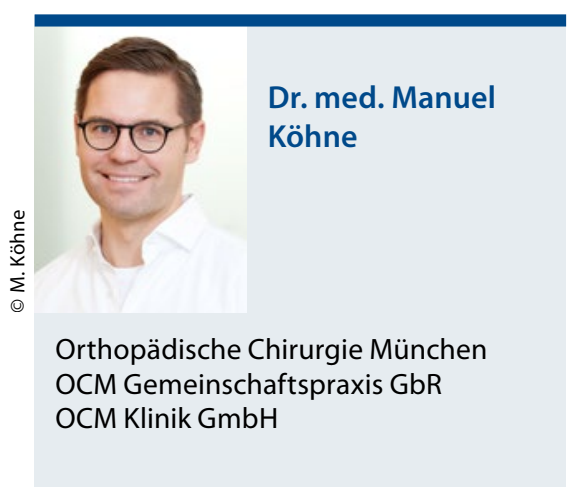

eine Mindestmenge an Knieendoprothesen pro Betriebsstätte (Krankenhaus) pro Jahr gefordert. Seit Oktober 2012 können sich Medizinische Einrichtungen als EndoProthetikZentrum (EPZ) und als EndoProthetikZentrum der Maximalversorgung (EPZmax) zertifizieren lassen, wenn sie die gestellten Anforderungen in einem Audit nachgewiesen haben. 2017 gibt es 524 Endocert-zentifizierte Kliniken in Deutschland. 